\title{
The Neuroprotective Effect of Carvedilol on Diabetic Neuropathy: An In Vitro Study
}

\author{
Rania M. Magadmi $\mathbb{D}^{1,2}$ Mujahid A. Alsulaimani, ${ }^{1,3}$ Aziza Rashed Al-Rafiah $\mathbb{D}^{1,4}$ \\ and Ahmed Esmat iD 1,5 \\ ${ }^{1}$ Department of Pharmacology, Faculty of Medicine, King Abdulaziz University, Jeddah, Saudi Arabia \\ ${ }^{2}$ Neuroscience Unit, Faculty of Medicine, King Abdulaziz University, Jeddah, Saudi Arabia \\ ${ }^{3}$ Department of Pharmacy, Ministry of Health, Taif, Saudi Arabia \\ ${ }^{4}$ Medical Laboratory Technology Department, Faculty of Applied Medical Sciences, King Abdulaziz University, Saudi Arabia \\ ${ }^{5}$ Department of Pharmacology and Toxicology, Faculty of Pharmacy, Ain Shams University, Cairo, Egypt
}

Correspondence should be addressed to Rania M. Magadmi; rmagadmi@kau.edu.sa

Received 18 May 2020; Revised 23 December 2020; Accepted 6 January 2021; Published 18 January 2021

Academic Editor: Antonio Brunetti

Copyright (c) 2021 Rania M. Magadmi et al. This is an open access article distributed under the Creative Commons Attribution License, which permits unrestricted use, distribution, and reproduction in any medium, provided the original work is properly cited.

\begin{abstract}
Diabetic neuropathy serves as a major complication for diabetic patients across the world. The use of effective treatment is integral for reducing the health complications for diabetic patients. This study has evaluated the carvedilol potential neuroprotective effect on diabetic neuropathy. An in vitro model of diabetic neuropathy was used, including dorsal root ganglia (DRG) that were cultured from male adult mice C57BL. These were incubated for about twenty-four hours in high glucose (HG) media (45 mM). Some cells were incubated with carvedilol $(10 \mu \mathrm{M})$. Neuronal viability, neuronal morphology, and activating transcription factor 3 (AFT3) were measured. The cell viability was decreased, along with neuronal length, soma area, and soma perimeter with HG media. Also, there was an overexpression of ATF3, which is a neuronal stress response marker. The pretreatment with carvedilol increased the viability of DRG as compared to HG-treated cells. Also, it significantly protected the DRG from HG-induced morphology changes. Though it shows a decrease in AFT3 expression, the statistical results were insignificant. The current study demonstrates the neuroprotective effect of carvedilol against HG-induced DN using an in vitro model. This could be through carvedilol antioxidant effects.
\end{abstract}

\section{Introduction}

The prevalence of diabetic neuropathy (DN) is recognized to affect about 50 percent of diabetic patients (both type 1 and type 2). Most studies confirm the DN as the major reason for diabetic patient's hospitalization as well as nontraumatic amputations [1]. Callaghan et al. [2] state that the management of DN is generally attained through appropriate blood glucose levels within the normal range and symptomatic therapy. Although various researches have explored DN chronic pain management, only a few improvements are achieved, where the remaining fail to meet the clinical needs of DN [3]. Tesfaye [4] noted that only two compounds show a clinical improvement, namely, $\alpha$-lipoic acid and epalrestat. Tesfaye [4] and Kumar and
Mitta [3] stated that effective medications need to be developed to prevent DN progression.

Carvedilol is an $\alpha$ and $\beta$ adrenoreceptors blocker and has peripheral vasodilators effect by blocking the $\alpha 1$ adrenoreceptor $[5,6]$. It is often prescribed for the treatment of chronic heart failure, hypertension, and left ventricle dysfunction after myocardial infarction. It also helps to block the oxygen radicals to damage nitric oxide-mediated coronary artery vasodilation [7]. Liu and Wang [8] and Diogo et al. [9] further emphasized the neuroprotective effects of carvedilol due to its antioxidant property. Areti et al. [10] further show the tendency of carvedilol to counteract the oxaliplatin-induced oxidative stress in neuronal cells.

These properties of the carvedilol make it suitable for use in the therapeutic treatment of DN patients. Thereby, this 
study is aimed at investigating the potential protective effect of carvedilol using in vitro model of $\mathrm{DN}$.

\section{Material and Methods}

2.1. Ethical Approval. This study followed all ethical guidelines related to the handling of animals and in vitro procedures. Also, the ethical committee at the Faculty of Medicine, at King Abdulaziz University (Reference No. 237-18), approved this study. The researcher ensured the practice of procedures that minimized the suffering of the animals.

2.2. DRG Isolation and Culture. Primary adult dorsal root ganglion (DRG) cultures were used as in vitro model of sensory neurons. DRGs were isolated from male adult C57BL mice. Cervical dislocation led to the killing of the mice, where the spinal column was cut into halve, at the midline. The spinal column was isolated and put on the phosphate buffer saline (PBS) in the petri dish on ice. The DRGs were collected on PBS. After removing the attached fibers from DRGs, DRGs were incubated with digestive enzymes $(0.06 \mu \mathrm{g} / \mathrm{ml}$ of collagenase XI (Sigma) and $0.1 \mu \mathrm{g} / \mathrm{ml}$ of dispase (Sigma) in PBS) at $37^{\circ} \mathrm{C}$ for one hour, $5 \% \mathrm{CO}_{2}$. Following it, pipetting was held for triturating the DRG. For neuron selective isolation, a gradient centrifuge technique with $15 \%$ bovine serum albumin (BSA) in the medium was used. These were then suspended in neurobasal A media (NBA; Gibco) (with $25 \mathrm{mM}$ glucose) supplemented with $2 \mathrm{mM}$ Glutamax (Gibco), 1\% Penicillin/Streptomycin (Gibco), and 2\% B-27 supplement (Gibco).

The poly-D-lysine (Sigma, $0.1 \mathrm{mg} / \mathrm{ml}$ ) was used for coating the well at the mid of the 12-coverslip well plate (or 96well plates). After this, the plate was incubated at $37^{\circ} \mathrm{C}, 5 \%$ $\mathrm{CO}_{2}$ for two hours. Following it, poly-D-lysine was removed, and the wells were washed with distal water (three times). Then, each well was added with laminin (Life Technologies, $10 \mu \mathrm{g} / \mathrm{ml}$ ) and further incubated for two hours. This laminin was then removed, following which the cells were placed at the center of each well and then incubated overnight.

2.3. Induction of In Vitro Models of Diabetes Neuropathy. The high glucose (HG) medium ( $45 \mathrm{mM}$ glucose) was used for the incubation of some DRG cells when the one day has passed after plating had been held for twenty-four hours [11, 12]. The duration of the experiment was decided based on the preliminary data results. The DRG cultures were exposed to HG concentration at different time points $(2,4,6$, and 24 hours), following which the cell viability is assessed (Figure 1). The DRG viability had substantially decreased after a day of being exposed to HG exposure to $72 \%$ $(p=0.0086)$. Thus, each group culture was incubated for 24 hours before the experiment.

The DRG culture was divided into 4 groups. The control group had standard medium glucose concentration for optimum neuron growth $(25 \mathrm{mM}), \mathrm{HG}$ group $(45 \mathrm{mM})$, and HG media $(45 \mathrm{mM})$ plus carvedilol (Roche, $10 \mu \mathrm{M})$ [10, 13]. Finally, the reference group has $(45 \mathrm{mM})$ medium plus $\alpha$ lipoic acid $(100 \mu \mathrm{M})[14]$. The carvedilol and lipoic acid were

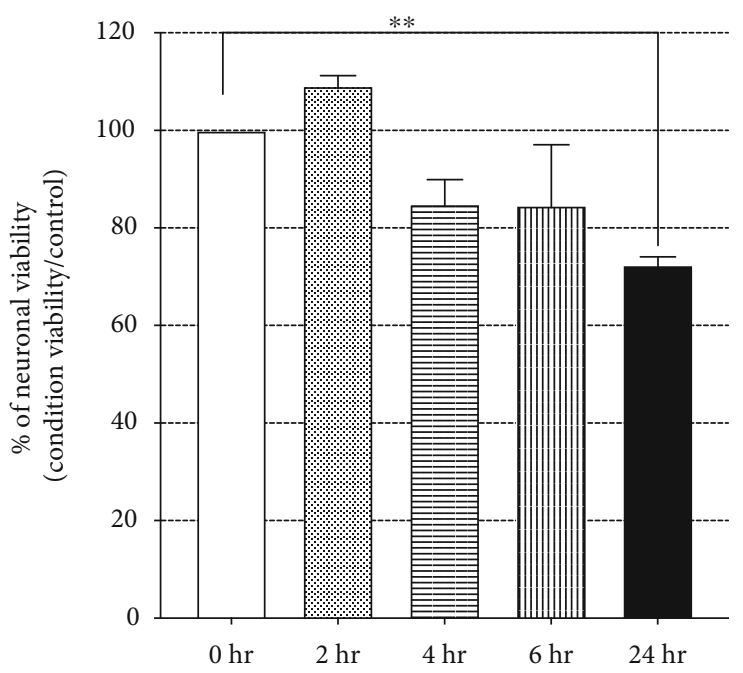

FIgURE 1: Effect of high glucose medium ( $45 \mathrm{mM})$ on DRG culture viability at different time points.

dissolved in dimethyl sulfoxide (DMSO). Then, the safety and efficacy of carvedilol were assessed as shown in Figure 2.

2.4. Viability Assay. Calcein-AM fluorescent assay kit was used for cell viability assessment (Vibrant cell assay kit, CAT\#V13181, Invitrogen, Life Technologies, USA). It is a nonfluorescent lipophilic dye. This is cleaved using the endogenous esterases to calcein, intracellularly, a highly green fluorescent (Figure 3). It is considered a viability maker because it is quenched upon the loss of cell viability. DRG cells were cultured in a 96-well plate with the corresponding drugs for 24 hours before performing the viability test. As per company protocol, $5 \mu \mathrm{m}$ of calcine was added to each well and incubated for 45 minutes at $37^{\circ} \mathrm{C}$ in the dark. Thereafter, cell viability was measured by a fluorescent microplate reader (BioTek ${ }^{\circledR}$, Synergy HT, USA) at excitation and emotion of 480/520, respectively. Experiments were run in triplication. Background fluorescence from DRG-only wells was subtracted. The viability was presented as the percentage of living cells to the control group.

2.5. Immunocytochemistry. DRG immunocytochemistry was performed using a 12-well plate. Phosphate buffer saline (PBS) was used to wash the cells thrice, after which these were incubated with $4 \%$ paraformaldehyde for ten minutes. Following it, paraformaldehyde was removed and PBS was used to wash the cells; Triton X-100 (Sigma Aldrich (MERCK), USA) (0.25\%) was used for enhancing the dye permeability across the cell membrane and left for ten minutes at room temperature. Then, the cells were incubated with blocking buffer ( $2 \%$ goat serum (Life Technologies, USA), $0.2 \%$ fish serum gelatine (Sigma, G7765), and $0.025 \%$ Triton X-100 in PBS for one hour at room temperature. These were then stained with primary antibody (mouse anti-B III tubulin monoclonal IgG; R\&D MAB1195 clone $\mathrm{TuJ}-1 ; 1: 1000$ in blocking buffer), overnight at $4^{\circ} \mathrm{C}$. Followed by the removal of the primary antibody, a blocking buffer was used to wash the cells thrice. The incubation of the cells was 


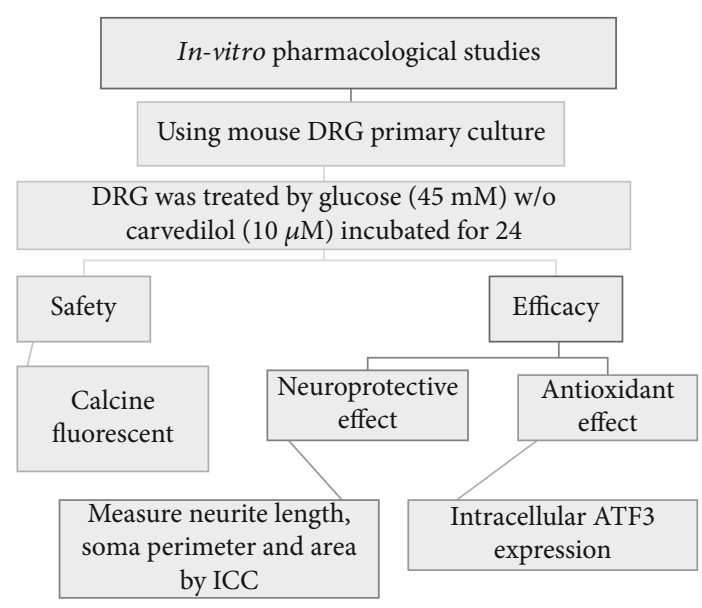

Figure 2: Procedure followed in this in vitro study.

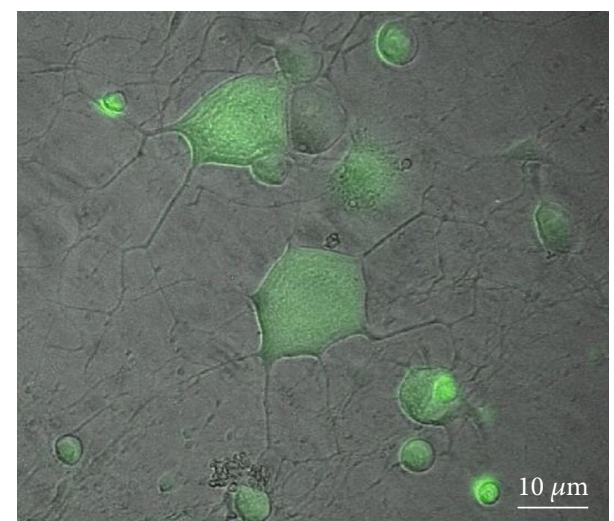

FIGURE 3: Calcein-AM-stained fluorescent image of the DRG culture.

held for secondary antibody Alexa Fluor ${ }^{\circledR} 594$ goat antimouse (Invitrogen A-21203, 1:1000) for two hours in the dark at room temperature. Following it, the removal of secondary antibodies was held with thrice washing with PBS. The cells' nuclei were stained by $4^{\prime}$, 6-diamidino-2-phenylindole (DAPI) (Invitrogen, P36935, USA). Each condition was presented with two coverslips, where the experiments were repeated thrice. $20 \mathrm{x}$ and $40 \mathrm{x}$ objectives of the fluorescent microscope (Nikon Y-TV55, Japan) were used for capturing images. The Fiji-ImageJ [15] software was used for image analysis.

2.6. DRG Morphology Analysis. Antitubulin III antibody was used for the staining of the DRG cells for detecting the neurons and neuritis. The ImageJ software was used for different random sections to measure the neurite length, soma area, and perimeter. Neurite length was measured by tracing each neurite to identify the total number of neurite length (in $\mu \mathrm{m}$ ), then divided over the number of neurons present in the section. The soma area and perimeter were measured by using the freehand selection tool around the edge of each neuron.

2.7. Evaluation of Neuronal Stress Response in DRG Culture. The expression of activation transcription factor 3 (ATF3)

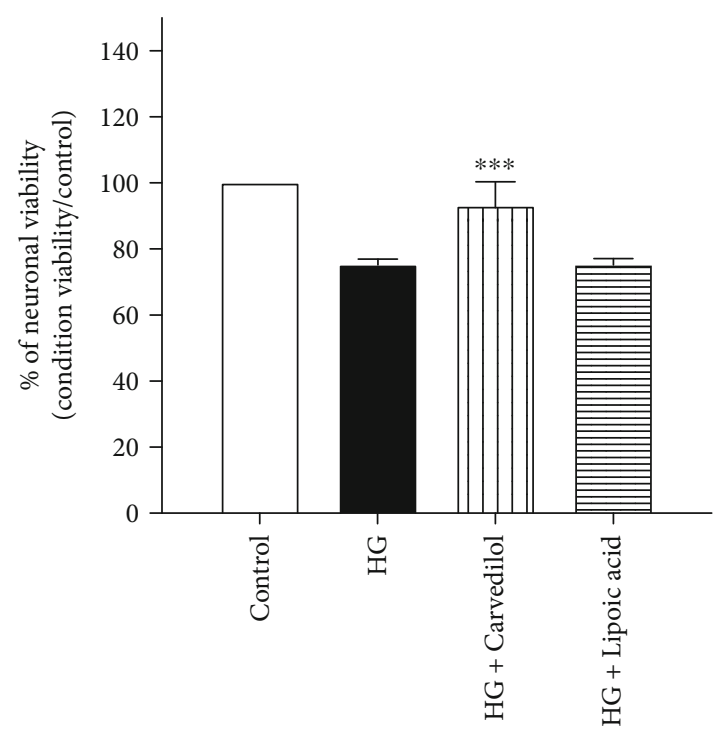

FIGURE 4: Carvedilol effect on HG-induced decreases in DRG viability.

was used for evaluating HG-induced stress in cultured DRG [16]. Conjugated ATF3 antibody (ATF3, Polyclonal, bs0519R, Bioss Antibodies, USA; 1:100) was used for cells staining. Fluorescent microscopy was used for imaging of cells at power of $20 \mathrm{X}$. These experiments were conducted in triplication. Negative control was used for non-treated cells. ATF3 percentages were calculated for positive neurons and compared between the groups.

2.8. Statistical Analysis. The findings were presented in the form of mean \pm standard error (SE). One-way Analysis of Variance (ANOVA) was used to make comparisons to the HG group. Each experiment was repeated three times in duplication. The GraphPad Prism version 8 software (GraphPad Software, La Jolla, CA, United States) was used for statistical analysis. The statistical significance was set at 0.05 .

\section{Results}

3.1. Effect of Carvedilol on HG-Induced Decreases in DRG Viability. In order to evaluate the neuroprotective effect of carvedilol in diabetic neuropathy, DRG cultures with HG media were incubated with or without carvedilol. Figure 4 shows the percentage of cell viability between different groups. The percentage of viability was calculated relative to negative control as $100 \%$ viability. The DRGs exposed to HG media showed more than $20 \%$ significantly decreased cell viability compared to the corresponding control $(p=0.0001)$. The neurons in HG media were found to be $75 \%$ viable using a calcein live-dead viability kit. However, $10 \mu \mathrm{M}$ of carvedilol significantly increased the DRG viability by more than $18 \%$ compared to the HG group $(p=0.001)$.

3.2. Effect of Carvedilol on Neuronal Morphology in HGExposed DRG. To investigate the protective effect of carvedilol on neuronal morphology, DRG cultures were incubated with HG with and without carvedilol. Then, cultures were 

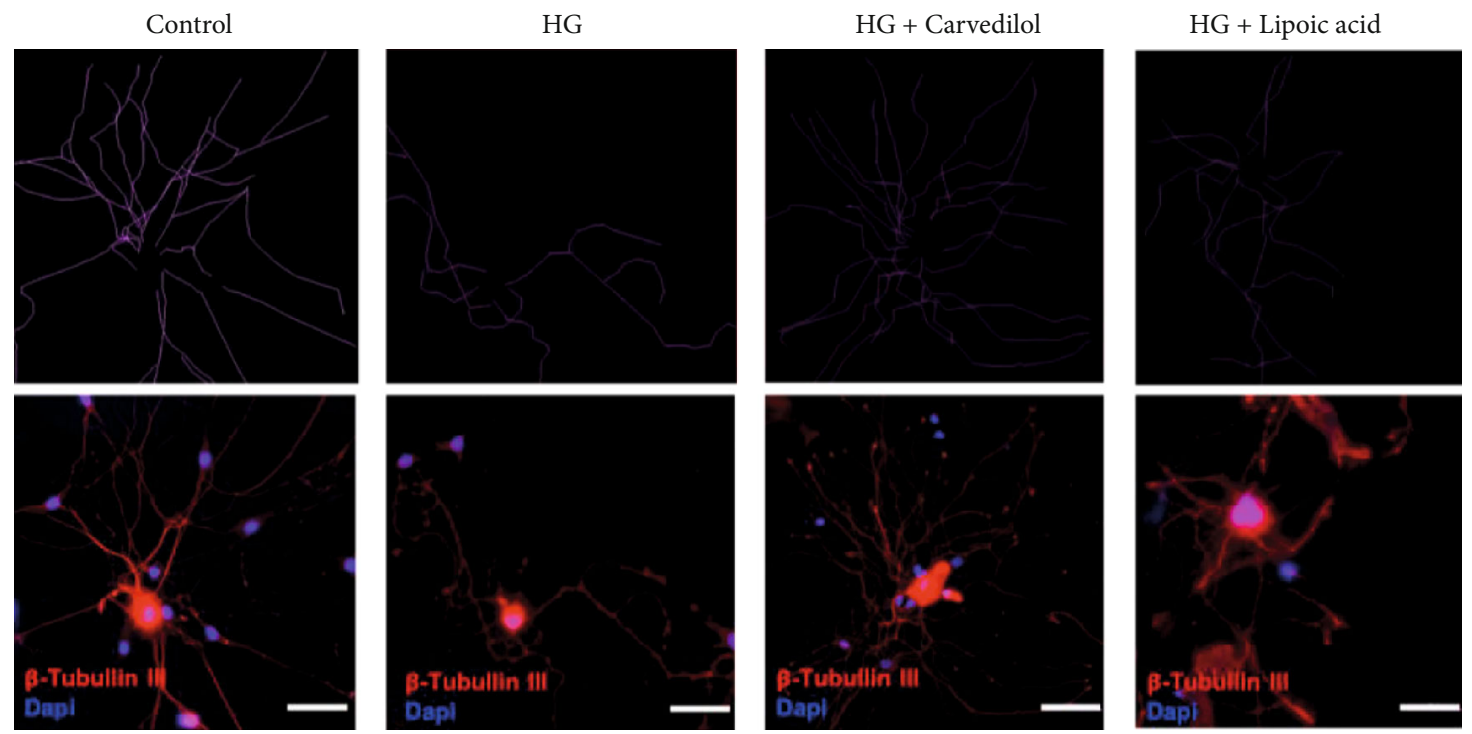

(a)

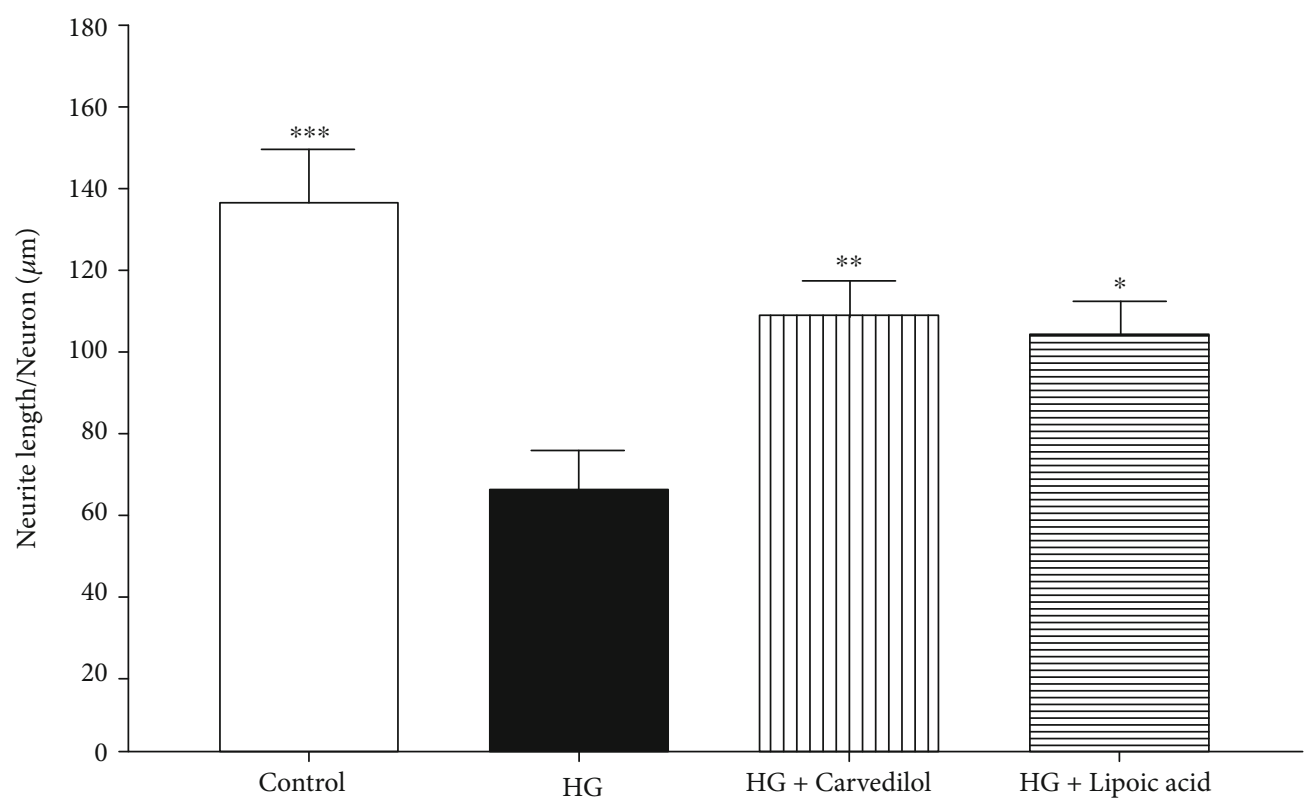

(b)

FIgURE 5: Effect of the carvedilol on neurite length in high-glucose media.

stained with anti- $\beta$ III tubulin $\mathrm{Ab}$ to visualize the neurons and neurites. Finally, the neuron perimeter, area, and neurite length were measured.

3.3. Neurite Length. As shown in Figure 5, the DRG exposed to HG for 24 hours showed a significant reduction in neurite length compared to control cells $(65.08 \mu \mathrm{m}$ and $136.9 \mu \mathrm{m}$, respectively; $p<0.001)$. However, $10 \mu \mathrm{M}$ of carvedilol prevented HG-induced reduction in neurite outgrowth by about $60 \%$. Also, the $\alpha$-lipoic acid showed the same improvement effect on the neurite length.

3.4. Soma Perimeter. As shown in Figure 6, there was a significant decrease $(p<0.001)$ in the soma perimeter of the cells exposed to HG media compared to the corresponding con- trol (from $29.85 \mu \mathrm{m}$ to $22.78 \mu \mathrm{m}$ ). Carvedilol $(10 \mu \mathrm{M}$ ) was able to improve the neuronal perimeter by $58 \%$ compared to the HG group. Interestingly, $\alpha$-lipoic acid has the same effect on neuronal perimeter shown in the cells treated with carvedilol.

3.5. Soma Area. As shown in Figure 7, the area of neurons was significantly decreased more than 1.5 -fold in response to HG $(p=0.02)$. In contrast, $10 \mu \mathrm{M}$ of carvedilol could protect the reduction in the area of the neurons by more than 2fold compared with high glucose $(p<0.001)$ and more than the control. Remarkably, lipoic acid improved the reduction of the soma area but did not reach a statistically significant level $(p=0.52)$. 


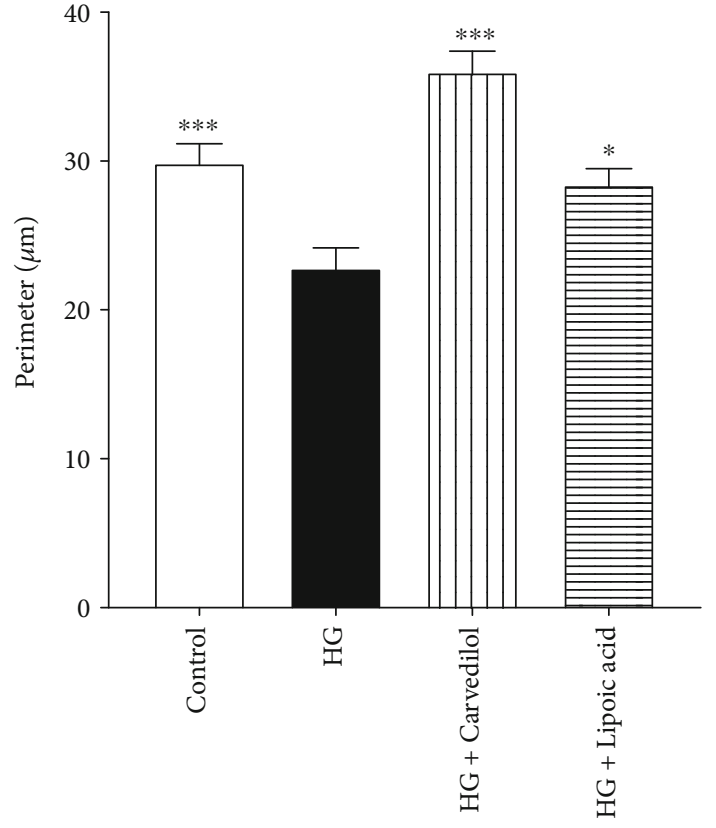

Figure 6: Effect of the carvedilol on soma perimeter in high-glucose media.

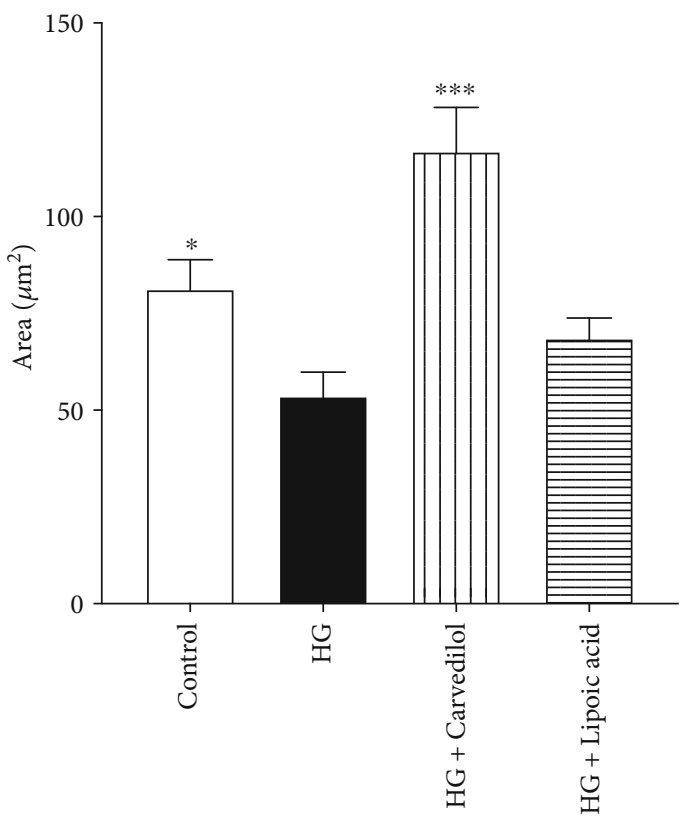

Figure 7: Effect of the carvedilol on soma area in high-glucose media.

Taken together, these results demonstrate that carvedilol could protect against morphological changes induced by HG in neuronal cells.

3.6. Effect of Carvedilol on ATF3 Expression on Neurons. To investigate the mechanism of the neuroprotective effect of carvedilol, the neuronal stress marker (ATF3) expression was evaluated. $10 \mu \mathrm{M}$ of carvedilol decreased the ATF3 expression in HG-treated DRGs, but this did not achieve statistical significance $(p=0.115)$ as shown in Figure 8 .

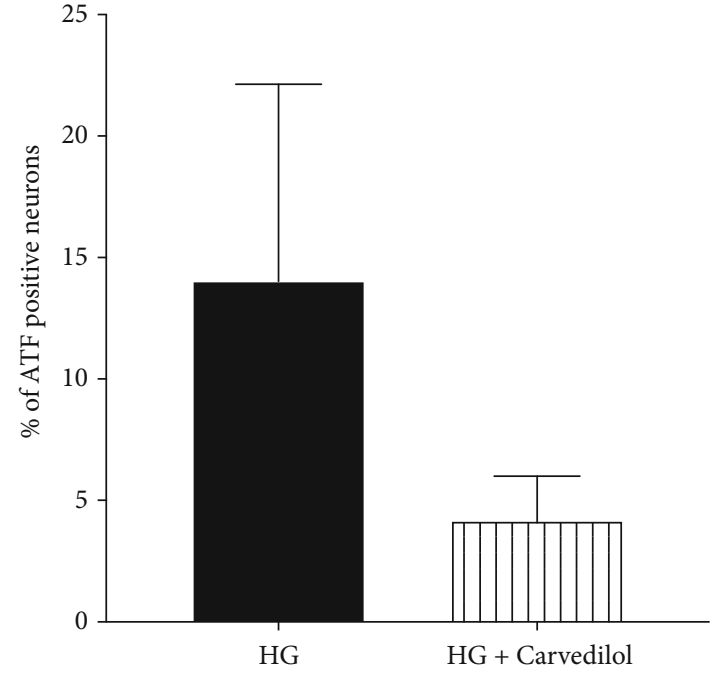

FIgURE 8: Percentage of ATF-positive neurons in high-glucose DRG cultures.

\section{Discussion}

Diabetes mellitus affects around 463 million people worldwide and is the most common cause of neuropathy. Several mechanisms could explain the pathogenesis of hyperglycemia to induce microvascular complications in diabetic patients. Oxidative stress could play an important role in cellular injury [17]. Recently, carvedilol has also been appreciated for its antioxidant activity [16, 18-20]. Moreover, diabetes and hypertension are common comorbidities specialty in advanced age patients [21]. The incidence of hypertension increases among diabetic patients [22] and vice versa [23]. Taken together, those attractive properties of carvedilol make it a suitable therapeutic candidate for managing diabetic neuropathy. Therefore, the neuroprotective impact of carvedilol has been examined using the in vitro model of $\mathrm{DN}$.

The use of in vitro method was based on providing new insights. Such as according to Vincent et al. [14], the study of peripheral neuropathy and DN uses DRG extensively. According to Melli and Höke [24], DRG culturing could be cell lines. Melli and Höke [24] examined that the cell line is inferior as compared to the primary cell culture as it gives fewer stimuli of an in vivo biological method. Melli et al. [25] assessed the benefits of adult rodent DRG culturing as they do not require serum and NGF in their growth, unlike embryonic DRG culture.

In the current study, the neurite length and soma area and perimeter also decreased with the induction of HG media. Similar results in previous studies showed that the short-term exposure to HG media caused a reduction in the neurite outgrowth in primary DRG cultures [26]. They contributed this effect as the increase of reactive oxygen species (ROS) generation and the decrease in the GSH level [26]. An increase in ROS generations after exposed to HG concentration is time-dependent [26]. An increase in ROS and intracellular Ca regulate and release the proapoptotic factors leading to altering the neuron morphology and subsequently cells death [26]. Moreover, HG induces swelling 
in mitochondria depolarization in sensory neurons leading to depletion in ATP and subsequently apoptosis. Thus, the oxidative stress represents a target for the neuroprotective effect of therapeutic intervention in diabetic neuropathy. Therefore, carvedilol was an attractive candidate to prevent diabetic neuropathies.

In the current study, carvedilol significantly improved neuronal survival and neurite length in HG media. Consistent with these results, a previous study showed that carvedilol concentration from 0.1 to $20 \mu \mathrm{M}$ significantly protects cortical neuronal cultures from cytotoxicity during hypoxia and reoxygenation model [27]. Moreover, $10 \mu \mathrm{M}$ of carvedilol prevents oxaliplatin-induced neurotoxicity in a neuronal cell line $(\mathrm{N} 2 \mathrm{a})$ by significantly reduced ROS production [10]. However, no previous in vitro work has been done to evaluate the carvedilol effect in the diabetic neuropathy model. To the best of our knowledge, this is the first in vitro experiment to demonstrate the neuroprotective effect of carvedilol in diabetes neuropathy.

Remarkably, carvedilol in this study increased the soma diameter and perimeter in HG media even more than the control group. This could be explained by understanding the heterogenicity nature of DRG cultures. DRG contains 3 subtypes of sensory neurons: $A \beta$-fibers, $A \delta$-fibers, and Cfibers. These fibers are classified based on their conduction velocity which positively correlates with the diameter and degree of myelination. The largest diameter fibers with thick myelination are $\mathrm{A} \beta$-fibers. They have the fastest conduction velocity, and they are classified as nonnociceptive neurons as they respond only to innocuous stimuli such as light touch. $A \delta$ - and c-fibers are nociceptive neurons with medium and small diameters, respectively $[28,29]$. Based on the results from the current study, one can conclude that carvedilol protects the nonnociceptive fibers more than nociceptive fibers. Subsequently, the average of soma diameter and perimeter increased compared to the normal heterogenous population of the control group. However, more immunostaining experiments to visualize the subtypes of preserved neurons in DRG must be carried out to confirm this speculation. Also, the clinical correlation must be considered.

Although the effect of carvedilol on HG-induced oxidative stress in DRG was not directly investigated in this study, the expression of ATF3 was used as a marker of neuronal stress [16, 30, 31]. Wright et al. [31] reported the increase in the expression of ATF3 in sensory neurons in both an in vitro and in vivo diabetic model. The current study showed that $10 \mu \mathrm{M}$ of carvedilol reduced, although not reached the statistically significant level, the expression of AFT3 in DRG exposed to HG. This nonsignificant finding could be explained by the relatively small number of neuronal cells in primary DRG cultures. More repeated experiments may be needed to draw a clear conclusion.

Carvedilol exerts antioxidant activity in two distinct ways. These mechanisms can be divided into chemical and biological aspects. Carvedilol could bind chemically and scavenge ROS molecules. Biologically, carvedilol could inhibit the enzymes that generate the ROS, leading to a marked decrease in the ROS production [20]. Evidence from the study supplements the therapeutic efficacy of carvedilol due to its antioxidant and anti-inflammatory activities. Liu and Wang [8] also note the neuroprotective properties of carvedilol, such as it shows improved results for the carvedilol protection of neurons against death.

On the contrary, carvedilol can cover the tachycardia, just like other adrenoreceptor blockers that serve as a warning indication for insulin-induced hypoglycemia in diabetic patients. Likewise, the risk of hypoglycemia and hypoglycemia unawareness can be elevated with the use of betablockers by patients with diabetes. However, there is minimal evidence for supporting the establishment that beta-blockers can be routinely contraindicated in diabetes since they have few clinically essential effects on hypoglycemia recovery and unawareness. Symptoms such as sweating might be improved even though some hypoglycemia signs including palpitation and tremor might be blunted [32-35]. The reason is that sweating is a sympathetic cholinergic reaction to hypoglycemia, which cannot be repressed by beta-blockers, and cautious use of beta-blockers improves sympathoadrenal activation by hypoglycemia. Additionally, the total signs of hypoglycemia might not be affected significantly by betablockers [36]. Moreover, some studies have recommended that beta-blockers have a minute effect on the risk of hypoglycemia and its risk, specifically from b1-selective betablockers. Thereby, diabetic patients should preferentially use selective beta-blockers with cardiovascular diseases not merely to enhance the long-term cardiovascular outcome, but also for potentially life-saving effects throughout severe hypoglycemia $[37,38]$.

\section{Conclusion}

This study presents the analysis of the neuroprotective effect of carvedilol against in vitro HG-induced neuronal damage. Although this study presents effective protection and antioxidant mechanism of carvedilol, more studies are needed so that the molecular mechanism of the antioxidant effect of carvedilol on sensory neurons as well as intracellular signaling can be established. Also, there is a need to establish a human clinical trial for assessing the effectiveness of carvedilol on DN. Since the study was carried out in Saudi Arabia, thereby, Saudi vision 2030 can derive valuable findings from this study, where a potential cure for DN can be discovered for supporting the national economy. Also, the findings could help reduce the mortality, as well as morbidity related to $\mathrm{DN}$ along with its related health care expenses. Additional studies are required for evaluating the advantages and disadvantages of beta-blockers for patients with diabetes.

\section{Data Availability}

The datasets used and analyzed during the current study are available from the corresponding author on reasonable request.

\section{Conflicts of Interest}

The author declares that there is no conflict of interest regarding the publication of this paper. 


\section{Acknowledgments}

The authors thank the Deanship of Scientific Research (DSR) for technical and financial support. The authors would like to thank Dr. Kariman Borouk for her help in conducting the experiments. This project was funded by the Deanship of Scientific Research (DSR) at King Abdulaziz University, Jeddah, under grant no. G. 342-248-1439. The authors, therefore, acknowledge with thanks DSR for technical and financial support.

\section{References}

[1] N. J. Shatnawi, N. A. Al-Zoubi, H. M. Hawamdeh, Y. S. Khader, K. Gharaibeh, and H. A. Heis, "Predictors of major lower limb amputation in type 2 diabetic patients referred for hospital care with diabetic foot syndrome," Diabetes, metabolic syndrome and obesity : targets and therapy, vol. 11, pp. 313319, 2018.

[2] B. C. Callaghan, A. A. Little, E. L. Feldman, R. A. Hughes, and Cochrane Neuromuscular Group, "Enhanced glucose control for preventing and treating diabetic neuropathy," Cochrane Database of Systematic Reviews, vol. 6, 2012.

[3] A. Kumar and R. Mittal, "Nrf2: a potential therapeutic target for diabetic neuropathy," Inflammopharmacology, vol. 25, no. 4, pp. 393-402, 2017.

[4] S. Tesfaye, "Recent advances in the management of diabetic distal symmetrical polyneuropathy," Journal of Diabetes Investigation, vol. 2, no. 1, pp. 33-42, 2011.

[5] R. R. Ruffolo, M. Gellai, J. P. Hieble, R. N. Willette, and A. J. Nichols, "The pharmacology of carvedilol," European Journal of Clinical Pharmacology, vol. 38, Supplement 2, pp. S82S88, 1990.

[6] J. Nichols, A. C. Sulpizio, D. J. Ashton, P. Hieble, and R. R. Ruffolo, Jr, "In vitro pharmacologic profile of the novel betaadrenoceptor antagonist and vasodilator, carvedilol," Pharmacology, vol. 39, no. 5, pp. 327-336, 1989.

[7] G. Z. Feuerstein, G. Poste, and R. R. J. Ruffolo, "Carvedilol update III: rationale for use in congestive heart failure," Drugs of Today, vol. 31, no. 5, p. 307, 1995.

[8] J. Liu and M. Wang, "Carvedilol protection against endogenous $\mathrm{A} \beta$-induced neurotoxicity in N2a cells," Cell Stress and Chaperones, vol. 23, no. 4, pp. 695-702, 2018.

[9] C. V. Diogo, C. M. Deus, M. Lebiedzinska-Arciszewska, A. Wojtala, M. R. Wieckowski, and P. J. Oliveira, "Carvedilol and antioxidant proteins in a type I diabetes animal model," European Journal of Clinical Investigation, vol. 47, no. 1, pp. 19-29, 2017.

[10] A. Areti, P. Komirishetty, and A. Kumar, "Carvedilol prevents functional deficits in peripheral nerve mitochondria of rats with oxaliplatin-evoked painful peripheral neuropathy," Toxicology and Applied Pharmacology, vol. 322, pp. 97-103, 2017.

[11] X. Xu, H. Jiang, H. Liu, W. Zhang, X. Xu, and Z. Li, "The effects of galanin on dorsal root ganglion neurons with high glucose treatment in vitro," Brain Research Bulletin, vol. 87, no. 1, pp. 85-93, 2012.

[12] Y. P. Zhang, S. Y. Liu, Q. Y. Sun, J. Ren, H. X. Liu, and H. Li, "Proanthocyanidin B2 attenuates high-glucose-induced neurotoxicity of dorsal root ganglion neurons through the PI3K/Akt signaling pathway," Neural Regeneration Research, vol. 13, no. 9, pp. 1628-1636, 2018.
[13] L. Wang, R. Wang, M. Jin et al., "Carvedilol attenuates 6hydroxydopamine-induced cell death in PC12 cells: involvement of Akt and Nrf2/ARE pathways," Neurochemical Research, vol. 39, no. 9, pp. 1733-1740, 2014.

[14] M. Vincent, J. W. Russell, K. A. Sullivan et al., "SOD2 protects neurons from injury in cell culture and animal models of diabetic neuropathy," Experimental Neurology, vol. 208, no. 2, pp. 216-227, 2007.

[15] A. Schneider, W. S. Rasband, and K. W. Eliceiri, "NIH image to Image): 25 years of image analysis," Nature Methods, vol. 9, no. 7, pp. 671-675, 2012.

[16] E. Peeraer, A. Van Lutsenborg, A. Verheyen, R. De Jongh, R. Nuydens, and T. F. Meert, "Pharmacological evaluation of rat dorsal root ganglion neurons as an in vitro model for diabetic neuropathy," Journal of pain research, vol. 4, pp. 55-65, 2011.

[17] M. J. Fowler, "Microvascular and macrovascular complications of diabetes," Clinical Diabetes, vol. 26, no. 2, pp. 77-82, 2008.

[18] H. H. Arab and M. M. El-Sawalhi, "Carvedilol alleviates adjuvant-induced arthritis and subcutaneous air pouch edema: modulation of oxidative stress and inflammatory mediators," Toxicology and Applied Pharmacology, vol. 268, no. 2, pp. 241-248, 2013.

[19] S. Yasar, J. Xia, W. Yao et al., "Antihypertensive drugs decrease risk of Alzheimer disease: Ginkgo Evaluation of Memory Study," Neurology, vol. 81, no. 10, pp. 896-903, 2013.

[20] P. Dandona, A. Aljada, and A. Bandyopadhyay, "Inflammation: the link between insulin resistance, obesity and diabetes," Trends in Immunology, vol. 25, no. 1, pp. 4-7, 2004.

[21] W. S. Aronow, "Hypertension and the older diabetic," Clinics in Geriatric Medicine, vol. 24, no. 3, pp. 489-501, 2008.

[22] J. R. Sowers, M. Epstein, and E. D. Frohlich, "Diabetes, hypertension, and cardiovascular disease," Hypertension, vol. 37, no. 4, pp. 1053-1059, 2001.

[23] T. W. Gress, F. J. Nieto, E. Shahar, M. R. Wofford, and F. L. Brancati, "Hypertension and antihypertensive therapy as risk factors for type 2 diabetes mellitus," New England Journal of Medicine, vol. 342, no. 13, pp. 905-912, 2000.

[24] G. Melli and A. Höke, "Dorsal root ganglia sensory neuronal cultures: a tool for drug discovery for peripheral neuropathies," Expert Opinion on Drug Discovery, vol. 4, no. 10, pp. 1035-1045, 2009.

[25] G. Melli, C. Jack, G. L. Lambrinos, M. Ringkamp, and A. Höke, "Erythropoietin protects sensory axons against paclitaxelinduced distal degeneration," Neurobiology of Disease, vol. 24, no. 3, pp. 525-530, 2006.

[26] J. N. Singh, G. Jain, and S. S. Sharma, "In vitro hyperglycemia enhances sodium currents in dorsal root ganglion neurons: an effect attenuated by carbamazepine," Neuroscience, vol. 232, pp. 64-73, 2013.

[27] K. YAMAGATA, S. ICHINOSE, and M. TAGAMI, “Amlodipine and carvedilol prevent cytotoxicity in cortical neurons isolated from stroke-prone spontaneously hypertensive rats," Hypertension Research, vol. 27, no. 4, pp. 271-282, 2004.

[28] S. P. Hunt and P. W. Mantyh, "The molecular dynamics of pain control," Nature Reviews Neuroscience, vol. 2, no. 2, pp. 83-91, 2001.

[29] I. Basbaum, D. M. Bautista, G. Scherrer, and D. Julius, "Cellular and molecular mechanisms of pain,” Cell, vol. 139, no. 2, pp. 267-284, 2009. 
[30] H. Tsujino, E. Kondo, T. Fukuoka et al., "Activating transcription factor 3 (ATF3) induction by axotomy in sensory and motoneurons: a novel neuronal marker of nerve injury," Molecular and Cellular Neuroscience, vol. 15, no. 2, pp. 170$182,2000$.

[31] D. E. Wright, J. M. Ryals, K. E. McCarson, and J. A. Christianson, "Diabetes-induced expression of activating transcription factor 3 in mouse primary sensory neurons," Journal of the Peripheral Nervous System, vol. 9, no. 4, pp. 242-254, 2004.

[32] K. Dungan, J. Merrill, C. Long, and P. Binkley, "Effect of beta blocker use and type on hypoglycemia risk among hospitalized insulin requiring patients," Cardiovascular Diabetology, vol. 18 , no. $1,2019$.

[33] V. Chandrasekaran, K. Subramanian, and A. Muthuramalingam, "Propranolol abuse: a case report on the harmful consequence of over-the-counter medications," Indian Journal of Psychological Medicine, vol. 42, no. 4, pp. 394-396, 2020.

[34] T. Tsujimoto, T. Sugiyama, M. F. Shapiro, M. Noda, and H. Kajio, "Risk of cardiovascular events in patients with diabetes mellitus on $\beta$-blockers," Hypertension, vol. 70, no. 1 , pp. 103-110, 2017.

[35] R. Farhat, G. Su, A.-S. Sejling, N. Knight, S. J. Fisher, and O. Chan, "Carvedilol prevents counterregulatory failure and impaired hypoglycaemia awareness in non-diabetic recurrently hypoglycaemic rats," Diabetologia, vol. 62 , no. 4, pp. 676-686, 2019.

[36] X. Li, S. Yu, Z. Zhang et al., "Predictive modeling of hypoglycemia for clinical decision support in evaluating outpatients with diabetes mellitus," Current Medical Research and Opinion, vol. 35, no. 11, pp. 1885-1891, 2019.

[37] V. W. Zhong, J. Juhaeri, S. R. Cole et al., " $\mathrm{HbA}_{1 \mathrm{C}}$ variability and hypoglycemia hospitalization in adults with type 1 and type 2 diabetes: a nested case-control study," Journal of Diabetes and its Complications, vol. 32, no. 2, pp. 203-209, 2018.

[38] SPIRITS-J Study Investigators, H. Ohmura, T. Mita et al., "Real-world data on the incidence of macrovascular complications in Japanese patients with type 2 diabetes: the sitagliptin registration type 2 diabetes-Juntendo Collaborating Project," Diabetes Therapy, vol. 10, no. 3, pp. 1099-1111, 2019. 